\title{
UK Biobank
}

3 Beate Leppert ${ }^{1}$, Louise AC Millard ${ }^{1,2}$, Lucy Riglin ${ }^{3}$, George Davey Smith ${ }^{1}$, Anita

$4 \quad$ Thapar $^{3}$, Kate Tilling ${ }^{1}$, Esther Walton ${ }^{1,4 *}$, Evie Stergiakouli ${ }^{1,5 *}$

51 MRC Integrative Epidemiology Unit (IEU), Bristol Medical School (Population

$6 \quad$ Health Sciences), University of Bristol, Bristol, UK

$7 \quad 2 \quad$ Intelligent Systems Laboratory, University of Bristol, Bristol, UK

83 MRC Centre for Neuropsychiatric Genetics and Genomics, Cardiff University,

$9 \quad$ Cardiff, UK

104 Department of Psychology, University of Bath, Bath, UK

115 School of Oral and Dental Sciences, University of Bristol, Bristol, UK

*authors contributed equally to this work

Corresponding author:

Dr. Beate Leppert,

MRC Integrated Epidemiology Unit, University of Bristol, Oakfield House, Oakfield

Grove, Bristol BS8 2BN, UK

E-mail: beate.leppert@bristol.ac.uk

Short title: Cross-disorder MR-pheWAS of 5 major psychiatric disorders in UK

Biobank 


\section{ABSTRACT}

Psychiatric disorders are highly heritable and associated with a wide variety of social adversity and physical health problems. Using genetic liability (rather than phenotypic measures of disease) as a proxy for psychiatric disease risk can be a useful alternative for research questions that would traditionally require large cohort studies with longterm follow up.

Here we conducted a hypothesis-free phenome-wide association study in about 300,000 participants from the UK Biobank to examine associations of polygenic risk scores (PRS) for five psychiatric disorders (major depression (MDD), bipolar disorder (BP), schizophrenia (SCZ), attention-deficit/ hyperactivity disorder (ADHD) and autism spectrum disorder (ASD)) with 23,004 outcomes in UK Biobank, using the opensource PHESANT software package.

There was evidence after multiple testing $\left(p<2.55 \times 10^{-06}\right)$ for associations of PRSs with 226 outcomes, most of them attributed to associations of $P_{R S} S_{M D D}(n=120)$ with mental health factors and PRS ${ }_{A D H D}(n=77)$ with socio-demographic factors. Among others, we found strong evidence of associations between a 1 standard deviation increase in PRS $_{\mathrm{ADHD}}$ with 1.1 months younger age at first sexual intercourse $[95 \%$ confidence interval [CI]: $-1.26,-0.94] ; P^{2} S_{A S D}$ with $0.01 \%$ reduced lower erythrocyte distribution width [95\% $\mathrm{Cl}:-0.013,-0.007]$; $\mathrm{PRS}_{\mathrm{SCZ}}$ with 0.98 odds of playing computer games [95\%Cl:0.976,0.989]; $\mathrm{PRS}_{\mathrm{MDD}}$ with a 0.11 points higher neuroticism score [95\%Cl:0.094,0.118] and $\mathrm{PRS}_{\mathrm{BP}}$ with 1.04 higher odds of having a university degree [95\%Cl:1.033,1.048].

We were able to show that genetic liabilities for five major psychiatric disorders associate with long-term aspects of adult life, including socio-demographic factors, mental and physical health. This is evident even in individuals from the general population who do not necessarily present with a psychiatric disorder diagnosis. 


\section{AUTHOR SUMMARY}

51

52

Psychiatric disorders are associated with a wide range of adverse health, social and economic problems. Our study investigates the association of genetic risk for five common psychiatric disorders with socio-demographics, lifestyle and health of about 330,000 participants in the UK Biobank using a systematic, hypothesis-free approach. We found that genetic risk for attention deficit/hyperactivity disorder (ADHD) and bipolar disorder were most strongly associated with lifestyle factors, such as time of first sexual intercourse and educational attainment. Genetic risks for autism spectrum disorder and schizophrenia were associated with altered blood cell counts and time playing computer games, respectively. Increased genetic risk for depression was associated with other mental health outcomes such as neuroticism and irritability. In general, our results suggest that genetic risk for psychiatric disorders associates with a range of health and lifestyle traits that were measured in adulthood, in individuals from the general population who do not necessarily present with a psychiatric disorder diagnosis. However, it is important to note that these associations aren't necessary causal but can themselves be influenced by other factors, like socio-economic factors and selection into the cohort. The findings inform future hypotheses to be tested using causally informative designs. 


\section{INTRODUCTION}

69

Family and twin research as well as large-scale genome-wide association studies (GWAS) have shown that psychiatric disorders are highly heritable (1) and that genetic risks for psychiatric disorders also are associated with socio-economic factors, physical health outcomes as well as other psychiatric disorders (2-5). Using genetic liability (rather than phenotypic measures of disease) as a proxy for psychiatric disease risk can be a useful alternative for research questions that would traditionally require long-term follow up and big datasets due to the low prevalence of some of the psychiatric disorders of interest in the population (e.g. adult-onset health consequences of child neurodevelopmental disorders). In addition, while high genetic risk for a psychiatric disorder is not always indicative of a diagnosis of psychiatric disease, it can index underlying subthreshold symptomatology that can still impact later adversities and quality of life (6).

So far, studies have used hypothesis-driven approaches to investigate associations of genetic risk for psychiatric disorders with various psychiatric and health outcomes as well as lifestyle factors $(7,8)$. However, big data resources that are readily available, such as UK Biobank with about 500,000 participants, provide rich phenotypic information that can be used for hypothesis-free studies and offset the multiple testing burden. Phenome scans are a type of hypothesis-free analysis where the association of a trait of interest is systematically tested with a potentially large number of phenotypes and can be hypothesis-generating by identifying an association when there is no prior reason to expect that an association may exist. As all available phenotypes are tested and the less 'significant' results published alongside those of greater 'significance', phenome scans can help to reduce biases associated with 
hypothesis-driven studies where researchers might only publish the most desirable or expected results.

In a Mendelian randomization phenome-wide association study (MR-pheWAS) genetic risk is used as a proxy for lifelong liability for a disorder to explore associations of this genetic liability with traits that may evolve as a consequence. Understanding these associations will be essential to inform prevention or early intervention strategies. However, conclusions about causality are limited due to the low predictive power and high pleiotropic effects of genetic risk scores for psychiatric conditions (8).

The aim of this study was to investigate the associations between genetic risk for five common psychiatric disorders - attention-deficit/ hyperactivity disorder (ADHD), autism spectrum disorder (ASD), schizophrenia (SCZ), major depression (MDD) and bipolar disorder (BP) - with a wide range of socio-demographic, lifestyle, physical and mental health outcomes in UK Biobank, using the systematic hypothesis-free MRpheWAS approach. 
In total 334,976 participants of white British ancestry in UK Biobank were included in this study with an average age of 56 (standard deviation $[S D]=8$ ) years. A descriptive overview of selected UK Biobank study sample characteristics is given in Figure $1 \mathrm{~A}$. The UK Biobank participants are known to be more educated and healthier than the average UK population which is reflected in the high percentage of people with a university degree $(47 \%)$ and low prevalence of current smoking $(10 \%)$ in the sample, which is comparable to the full UK Biobank release (9). Furthermore, $34 \%$ of participants reported to have seen a general practitioner and $11 \%$ a psychiatrist for nerves, anxiety, tension or depression but there are few cases of schizophrenia $(n=132), \operatorname{ADHD}(n=71), \operatorname{ASD}(n=143)$ or bipolar disorder $(n=439)$. An overview of UK Biobank phenotype categories is given in Figure 1B.

\section{DISORDER SPECIFIC EFFECTS}

The MR-pheWAS of each psychiatric disorder tested the association of the respective polygenic risk score, aggregated from independent, genome-wide significant SNPs, with 23,004 outcomes in UK Biobank, adjusted for age, sex and the first 10 genetic principal components. There was strong evidence after multiple testing correction based on the number of independent tests derived from spectral decomposition $(p<2.55 \times 10-6)$ for associations of either the ADHD, ASD, SCZ, MDD or BP PRS with 226 outcomes in 31 UK Biobank categories (Figure 2 and Table S1) as described below. A less stringent 5\% FDR multiple testing threshold identified 209 additional outcomes also associated with at least one PRS (Figure S1). Correlations among the PRS can be found in supplementary Table S2. A detailed list of all MR-pheWAS results generated by the open-source PHESANT software package can be found in Table S3. 
130 Unless stated as a PHESANT result, estimates for continuous outcomes are generated by following up outcomes and manually curating the outcome phenotypes, to compute estimates on their original scale.

\section{Attention deficit/ hyperactivity disorder}

PRS $_{\text {ADHD }}$ was strongly associated with 77 outcomes (Figure 3) including 39 sociodemographic factors, 33 general health and 5 mental health, brain and cognition outcomes. The strongest evidence of association with $\mathrm{PRS}_{\mathrm{ADHD}}$ was seen for sociodemographic and lifestyle factors. A 1 SD higher $\mathrm{PRS}_{\mathrm{ADHD}}$ was associated with a 1.09 month younger age at first sexual intercourse [95\% confidence interval $[\mathrm{Cl}]$ : $-1.26,-0.94]\left(p=2.0 \times 10^{-16}\right)$, and 0.96 lower odds of having a university degree $[95 \% \mathrm{Cl}$ : and paternal age of their parents $\left(-0.08\right.$ years $[95 \% \mathrm{Cl}:-0.103,-0.051] p=4.4 \times 10^{-9} ;-0.10$ years [95\% Cl: $-0.134,-0.067] p=3.2 \times 10^{-9}$,respectively), 0.97 lower odds of average household income [95\% $\mathrm{Cl}: 0.96,0.97]\left(p=1.3 \times 10^{-20}\right), 1.05$ higher odds of current smoking $[95 \% \mathrm{Cl}: 1.03,1.06]\left(p=5.7 \times 10^{-15}\right)$ and 1.04 higher odds of experiencing physical abuse as a child $[95 \% \mathrm{Cl}: 1.02,1.06]\left(p=1.3 \times 10^{-6}\right)$.

Further, $1 \mathrm{SD}$ increase in $\mathrm{PRS}_{\mathrm{ADHD}}$ was associated with 17 physical health outcomes related to obesity, including $0.05 \mathrm{~kg} / \mathrm{m}^{2}$ higher BMI [95\%Cl: $\left.0.037,0.070\right]\left(p=7.4 \times 10^{-11}\right)$, leg and arm fat mass, waist circumference and trunk fat mass. Furthermore, there was

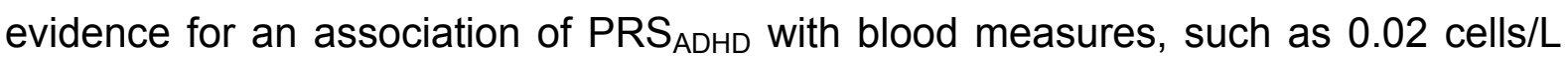
higher leukocyte count [95\%Cl: $0.012,0.026]\left(p=1.4 \times 10^{-7}\right)$.

Associations seen for brain and cognition include 0.04 points lower fluid intelligence score $[95 \% \mathrm{Cl}:-0.050,-0.026]\left(p=1.9 \times 10^{-9}\right)$. 


\section{Autism spectrum disorder}

155

156

PRS $_{\text {ASD }}$ was strongly associated with 9 outcomes (Figure 3), including 1 sociodemographic, 7 general health and 1 mental health outcome.

The strongest association of $\mathrm{PRS}_{\mathrm{ASD}}$ was found for lower erythrocyte distribution width where 1 SD higher PRS $_{\text {ASD }}$ was associated with $0.01 \%$ lower erythrocyte distribution width [95\% Cl: $-0.013,-0.007]\left(p=6.3 \times 10^{-10}\right)$ and 0.98 lower odds of comparative body size at age $10[95 \% \mathrm{Cl}: 0.97,0.98]\left(p=6.2 \times 10^{-11}\right)$. Furthermore, 1 SD higher $\mathrm{PRS}_{\mathrm{ASD}}$ was associated with $0.001 \mathrm{~g} / \mathrm{cm}^{2}$ lower heel bone mineral density (BMD) [95\%Cl:-0.002,$0.001]\left(p=4.1 \times 10^{-5}\right)$

The only mental health outcome that was associated with $\mathrm{PRS}_{\mathrm{ASD}}$ was 1.02 higher odds of being a nervous person ("suffer from nerves") [95\% Cl:1.01,1.03] $\left(p=7.7 \times 10^{-7}\right)$.

\section{Schizophrenia}

There was strong evidence of association for $\mathrm{PRS}_{\mathrm{SCZ}}$ with 11 outcomes (Figure 3), including 2 socio-demographic, 4 mental health and cognition and 5 general health outcomes.

The strongest evidence of an association with $\mathrm{PRS}_{\mathrm{SCZ}}$ was detected for playing computer games (OR:0.98 [95\%Cl:0.976,0.989] $\left.\mathrm{p}=1.4 \times 10^{-7}\right), 0.01 \%$ lower platelet distribution width $[95 \% \mathrm{Cl}: 0.003,0.006]\left(p=1.6 \times 10^{-7}\right)$ and having 0.68 lower odds of glioma cancer $[95 \% \mathrm{Cl}: 0.592,0.785]\left(p=1.2 \times 10^{-7}\right)$.

In addition, 1 SD increased $\mathrm{PRS}_{\mathrm{SCz}}$ was associated with $0.42 \mathrm{sec}$ longer duration of completing an online cognitive function test (alphanumeric path) [95\% $\mathrm{Cl}: 0.237,0.580]$ $\left(p=3.0 \times 10^{-6}\right)$ and a $19.1 \mathrm{~mm}^{3}$ reduced grey matter volume of the left putamen [95\%Cl:-26.9,-11.3] $\left(p=1.5 \times 10^{-6}\right)$. 


\section{Major depressive disorder}

$\mathrm{PRS}_{\mathrm{MDD}}$ was associated with 120 outcomes (Figure 3), including 18 sociodemographic, 76 mental health and 26 general health outcomes.

Most of the associations (63\%) were related to mental health, including an association of higher $\mathrm{PRS}_{\mathrm{MDD}}$ with higher odds of depression, anxiety, irritability, nervousness and mood swings. Strongest evidence of association with $P R S_{M D D}$ was found for 1.07 higher odds of "seen a doctor for nerves, anxiety, tension or depression" $[95 \% \mathrm{Cl}: 1.066,1.081] \quad\left(p=2.6 \times 10^{-81}\right), \quad 0.11$ points higher neuroticism score [95\%Cl:0.094,0.118] $\left(p=2.0 \times 10^{-16}\right)$ and 1.05 higher odds of having mood swings $[95 \% \mathrm{Cl}: 1.045,1.060]\left(p=1.8 \times 10^{-47}\right)$.

Furthermore, there was strong evidence of $1 \mathrm{SD}$ higher $\mathrm{PRS}_{\mathrm{MDD}}$ being associated with socio-demographic and lifestyle traits including 1.03 higher odds of ever smoking [95\%Cl:1.02,1.03] $\left(p=8.4 \times 10^{-14}\right)$ and 1.04 higher odds of cannabis use $[95 \% \mathrm{Cl}: 1.03,1.06]\left(p=2.3 \times 10^{-8}\right)$.

Associated physical health measures included 0.97 lower odds of taking medication for pain relief, constipation or heartburn [95\% Cl:0.967,0.981] $\left(p=4 \times 10^{-6}\right)$ and 1.02 odds of more frequent feelings of pain, e.g. back pain $[95 \% \mathrm{Cl}: 1.015,1031]\left(p=6 \times 10^{-9}\right)$.

\section{Bipolar disorder}

$\mathrm{PRS}_{\mathrm{BP}}$ was associated with 57 outcomes (Figure 3), including 19 socio-demographic, 35 general health and 3 mental health outcomes.

Socio-demographic and lifestyle factors included associations of higher $\mathrm{PRS}_{\mathrm{BP}}$ with 1.04 higher odds of having a university degree [95\% Cl:1.033,1.048] $\left(p=4.5 \times 10^{-26}\right)$, 0.02 hours/day less time spent watching television [95\% Cl:-0.027,-0.017] 
$\left(p=2.8 \times 10^{-15}\right)$ and lower average alcohol intake (OR:0.98 [95\%Cl:0.978,0.989] $\left.p=1.5 \times 10^{-7}\right)$.

General health traits included 19 traits indicating an association of $1 \mathrm{SD}$ higher $\mathrm{PRS}_{\mathrm{BP}}$ with $0.07 \mathrm{~kg} / \mathrm{m}^{2}$ lower body weight and fat mass [95\% $\left.\mathrm{Cl}:-0.090,-0.058\right]\left(\mathrm{p}=2.0 \times 10^{-16}\right)$ and 5 traits related to blood measures, such as $0.004 \%$ decreased platelet distribution width [95\%Cl:-0.006,-0.003] $\left(p=1.8 \times 10^{-6}\right)$.

The two traits related to mental health were risk taking (OR:1.03 [95\%Cl:1.018,1.035] $\left.p=2.9 \times 10^{-19}\right)$ and feeling fed-up (OR:0.98 $\left.[95 \% C l: 0.975,0.989] p=2.5 \times 10^{-7}\right)$.

\section{CROSS DISORDER CONSIDERATIONS}

210 The highest overlap of associated outcomes of the univariable MR-pheWAS scans was seen for ADHD and BP with 11 socio-economic and lifestyle and 12 general health outcomes associated with both disorders (Figure 4). However, the majority of the associations are directionally opposite for ADHD and BP. For example, higher $\mathrm{PRS}_{\mathrm{ADHD}}$ showed evidence for associations with lower educational attainment and higher $\mathrm{BMI}$, whereas higher $\mathrm{PRS}_{\mathrm{BP}}$ was associated with higher educational attainment and lower BMI. Only a higher risk of smoking initiation ("ever smoked") was directionally consistent for both $\mathrm{PRS}_{\mathrm{ADHD}}$ and $\mathrm{PRS}_{\mathrm{BP}}$.

Furthermore, all disorder PRSs showed some evidence for association with different blood cell counts, such as a decreased leukocyte count for $\mathrm{PRS}_{\mathrm{ADHD}}$ and $\mathrm{PRS} \mathrm{S}_{\mathrm{MDD}}$, or a decreased eosinophil count for $\mathrm{PRS}_{\mathrm{ADHD}}$ and $\mathrm{PRS}_{\mathrm{SCZ}}$.

221 There was very little overlap of highly associated outcomes between the neurodevelopmental domains (ADHD, ASD and SCZ). 


\section{SENSITIVITY ANALYSIS}

226 We repeated our tests of association of outcomes passing the spectral decomposition

227 threshold, additionally adjusting for additional potential confounders (assessment

228 centre, genotype chip and the first 40 principal components). Estimates were highly

229 consistent with our main results, as shown in Table S4.

230 Relaxing the p-value threshold for including SNPs in the PRS resulted in some

231 changes in the results (Figure S2). For ADHD, SCZ, MDD and BP the general trend

232 was an inflation of $p$-values, with more associations below the significance threshold

233 (Table S5) and higher effect estimates with smaller confidence intervals. A different

234 pattern was observed for autism spectrum disorder with inconsistent results for some

235 of the outcomes, as described in detail in the supplementary Text S1. Overall the

236 strength of associations obtained for blood cell count traits across disorders varied

237 between $p$-value thresholds, with weaker associations found for less stringent $p$-value 238 thresholds. 
In this study, we conducted a MR-pheWAS to examine the relationships between genetic liability for five major psychiatric disorders and 23,004 outcomes in about 300,000 UK Biobank participants.

Our results build on a large body of literature supporting links between genetic risk for psychiatric disorders with a wide variety of outcomes including psychological wellbeing, lifestyle, socio-demographic factors and physical health $(2,4,7,10,11)$. Our findings also suggest that although different psychiatric disorders show strong genetic overlap (7), genetic risk for distinct psychiatric disorders show differential associations with lifestyle, socio-demographic factors and physical health as highlighted in Figure 5. Genetic liability for ADHD and bipolar disorder showed the strongest associations with lifestyle and social environmental factors as well as physical health. On the other hand, genetic liability for major depression was most strongly associated with psychological health and associations with lifestyle and socio-demographic factors were less robust.

We were able to replicate previously reported associations between genetic liability for ADHD and lower educational attainment $(12,13)$, higher prevalence of smoking (14), younger age at delivery (15) and higher body mass index (16). While the previous findings for smoking and BMI were identified in young adults, our findings using an adult population-based sample with a mean age of 56 years, suggest that associations of childhood psychiatric disorder genetic liabilities with health and social outcomes persist into later adulthood. Associations of genetic liability for ADHD in childhood could represent effects of childhood ADHD or sub-threshold ADHD on long term social and economic outcomes, or alternatively associations could be due to parental effects 
(due to their shared genetic risk of ADHD) or horizontal pleiotropy (the same genetic variants affecting multiple traits).

Many of the associations of genetic liability for MDD with increased mood swings, irritability, feelings of loneliness and isolation are clinically known and have previously been reported (5). Our results are also in line with a recent publication from the Brainstorm consortium investigating genetic correlations among psychiatric disorders with neurological and quantitative traits using LD score regression and GWAS summary statistics, reporting high genetic correlations between most psychiatric disorders and educational attainment and BMI $(2,7)$. However, we found little evidence for associations of genetic liability for ADHD, ASD and schizophrenia with mental health outcomes, such as depressive symptoms, neuroticism or anxiety; and very few associations with cognitive or brain imaging outcomes, which might be because of the UK Biobank being a selected sample with lower rates of psychiatric disorders than the general population as discussed in the limitations section.

In addition to identifying previously reported associations of genetic liability for ASD, our MR-pheWAS also revealed novel associations. We found a strong association of genetic liability for ASD with decreased heel bone mineral density, which furthers previous evidence from observational studies that children and adolescents with ASD have lower bone mineral density $(17,18)$, higher frequency of bone fractures $(19)$ and lower vitamin D levels $(20,21)$, which is essential for bone metabolism. This might suggest that these observed associations may be due to pleiotropic effects of genetic variants associated with bone health. was associated with lower grey matter volume in the left putamen and a lower risk for 
glioblastoma cancer. Both phenotypes have been associated with schizophrenia in observational studies but it is not clear whether these phenotypes are determinants or consequences of schizophrenia, or due to confounding or shared genetic risk $(22,23)$. For glioblastoma our finding could be attributed to common underlying mechanisms that act in opposite directions, since it has been previously suggested that the same biological pathways leading to schizophrenia may be protective for developing glioblastoma (23). With respect to differences in brain volumes of schizophrenia patients, two large studies by the international ENIGMA (24) and Japanese COCORO (25) consortia found no notable difference in putamen volume between schizophrenia patients and controls or associations of brain volumes with a $\mathrm{PRS}_{\mathrm{SCZ}}$ in schizophrenia patients (26). Our results suggest that genetic risk for schizophrenia could be associated with putamen volume, but should be treated with caution because of potential selection bias due to the highly selected subset of about 10,000 UK biobank participants with brain scan data available at the time of data analysis (27). In line with our results, other previous work in schizophrenia patients and their relatives identified an association between schizophrenia and longer performance duration on the Trail Making Test (28), which requires searching and connecting irregularly arranged targets (digits and letters) in ascending order and is widely used to test for executive function, cognitive ability and processing speed (29-34).

Altered blood cell counts were associated with genetic liability for all disorders. Many psychiatric disorders previously have been associated with allergic or inflammatory states $(35-37)$, such as asthma $(38)$ and atopic diseases $(39,40)$ but it is unclear whether high inflammatory states are on the causal pathway to disorder manifestation or the result of comorbid and confounding behaviours associated with the disease, such as restricted diet, overweight, risky behaviours or medication. Our results support 
the possibility that altered blood cell counts could be a consequence of the disorder, but we cannot rule out contributions of horizontal pleiotropic effects. Also, considering the inconsistent findings from the sensitivity analyses for blood count traits, results need further validation and should be treated with caution.

\section{Limitations}

Patients with psychiatric disorders or high genetic liability for psychiatric disorders are known to be less likely to participate in studies in the first place and more likely to dropout during an ongoing study (41). Selection bias into a study as well as attrition can induce collider bias (42). There is consensus that the UK Biobank sample is not representative of the UK population, with participants showing, for example, lower prevalence of current smoking and lower rates of mortality (9). If both having a psychiatric disorder and a specific outcome (e.g. high socio-economic position) are associated with participation (the collider), this can induce an association between genetic risk for psychiatric disorders and the outcome, called collider bias. To reduce the possibility of collider bias we limited the set of included confounders in our main analysis but adjusted for assessment centre and genotype batch in sensitivity analysis.

A direct comparison of PHESANT estimates across the psychiatric disorders cannot be done without taking the differentially powered GWASs and derived PRS into account. This can also affect the number and set of outcomes associated with each disorder, which allows only a relative comparison among the outcomes. Further, the MDD GWAS used in the current study to calculate genetic risk scores included thirty thousand participants from UK Biobank (about $10 \%$ of the GWAS sample) which might have inflated our results for depression related items but is not expected to introduce bias in any other traits, such as blood counts. 
Although genetic risk scores were derived using variants associated at genome-wide significance level, they can still have horizontal pleiotropic effects on different disorders and traits. Hence, our reported associations cannot on its own inform about causality but should be followed up with other causally informative methods. We therefore encourage triangulation of results using other study designs $(43,44)$, such as two-sample MR, negative control or twin studies.

\section{Conclusion}

342 We were able to show that genetic liability for five common psychiatric disorders are associated with distinct aspects of adult life, including socio-demographic factors, mental and physical health. This is evident even in individuals from the general population who do not necessarily present with a psychiatric disorder diagnosis. However, there was surprisingly little overlap of findings for the different psychiatric

347 disorder genetic risk scores despite the high genetic and symptomatic overlap of psychiatric disorders, such as schizophrenia and bipolar disorder. Furthermore, we want to emphasize the benefit of using genetic instruments for hypothesis-generating efforts

in the field of psychiatry. 
METHODS

\section{Study population}

Between 2006-2010 UK Biobank recruited 503,325 men and women in the UK at ages

40-69 years. The cohort contains a large dataset including physical measurements, blood/urine/saliva samples, health and lifestyle questionnaires as well as genotype (https://www.ukbiobank.ac.uk/).

For 463,010 participants genotyping was performed using the Affymetrix UK BiLEVE Axiom array or Affymetrix UK Biobank Axiom® array. Participants with non-white British ancestry $(n=54,757)$ and 73,277 who have a kinship coefficient denoting a thirddegree relatedness were removed from an already quality checked dataset (excluding participants with withdrawn consent, sex mismatch or sex aneuploidy) (45), resulting in a dataset containing 334,976 participants (Figure 6).

UK Biobank received ethical approval from the research ethics committee (reference 13/NW/0382). All participants provided informed consent to participate. This work was done under application number 16729 (using genetic data version 2 [500K with HRC imputation] and phenotype dataset 21753).

\section{Polygenic risk scores}

Genetic variants were identified from the most recent GWAS summary statistics listed in Table 1 with $p<5 \times 10^{-8}$ for ADHD, ASD, SCZ, MDD and BP. This stringent $p$-value cut-off was chosen to minimize bias introduced by horizontal pleiotropic effects of genetic variants. All summary statistics were subject to standard quality control

372 including filtering for minor allele frequency (MAF>0.1) and imputation quality

373 (INFO>0.8) and excluding the MHC region on chromosome $6(26-33 \mathrm{Mb})$ due to its 
bioRxiv preprint doi: https://doi org/10.1101/634774; this version posted May 10,2019. The copyright holder for this preprint (which was not certified by peer review) is the author/funder, who has granted bioRxiv a license to display the preprint in perpetuity. It is made available under aCC-BY 4.0 International license.

374 complex linkage disequilibrium structure. Polygenic risk scores (PRS) were derived

375 using PRSice v2.13 by identifying independent risk alleles in approximate linkage

376 disequilibrium ( $\mathrm{R}^{2}<0.1$ within $500 \mathrm{~kb}$ distance) and computing a weighted, standardized

377 mean score from these, as has been described previously (46).

378 
Table 1. Details of GWAS used for calculating PRS

\begin{tabular}{lcccc} 
disorder & cases & controls & SNPs in PRS & Source \\
\hline ADHD & 20,183 & 35,191 & 10 & Demontis et al. (2019) (2) \\
ASD & 18,381 & 27,969 & 2 & Grove et al. (2017) (4) \\
Schizophrenia & 36,989 & 113,075 & 113 & Ripke et al. (2014) (3) \\
MDD & 135,458 & 344,901 & 34 & Wray et al. (2018) (5) \\
Bipolar disorder & 20,129 & 21,524 & 8 & Ruderfer et al. (2018)(47) \\
\hline
\end{tabular}

1- PRS derived from genome-wide significant hits $\left(\mathrm{p}<5 \times 10^{-8}\right)$

381 2- SNP heritability estimates reported in the corresponding discovery sample

382 ADHD - Attention deficit/hyperactivity disorder, MDD - Major depression, ASD - Autism spectrum disorder

\section{Outcomes}

UK Biobank provides a fully searchable data showcase

(http://biobank.ctsu.ox.ac.uk/crystal/ ) which at the time of data download (March

environment, socio-demographic, early life factors, anthropometry, family history and depression outcomes.

Age, sex and the first 10 principal components derived from the genetic data were

included as covariates in all regression models. Age was derived from the participants date of birth and the date of their first assessment centre visit. Sex was self-reported and validated using genetic data.

PHESANT package (version 0.17) was used to test the association of each PRS with each outcome variable in Biobank. A detailed description of PHESANT's automated rule-based method is given elsewhere $(48,49)$. In brief, decision rules are based on the variable field type and categorize each variable as one of four data types: 
400

401

402

403

404

405

406

407

408

409

410

411

412

413

414

415

416

417

418

419

420

421

422

423

variable (dependent) in a regression model, respectively. Normality of continuous data is ensured by an inverse normal rank transformation prior to testing. All estimates correspond to 1 SD change of the PRS. Selected continuous outcomes were followed up to compute meaningful estimates on the original phenotype scale for better interpretation.

PHESANT assigns each UK Biobank outcome to one of 91 level 3 categories based on the 235 origin categories of the UK Biobank catalogue (a full list of categories is provided in Table S1). Furthermore, three authors (BL, EW, ES) grouped these 91 categories into four prespecified higher level categories in order to aid result presentation: socio-demographics and lifestyle, brain and cognition, mental health and general health (Figure 1B).

To account for multiple testing ( $n=23,004$ tests) we used a previously derived threshold $(49,50)$ based on an estimate of the number of independent phenotypes calculated using spectral decomposition (phenoSPD) ( $n=19,645)$. The multiple testing adjusted significance threshold was $\mathrm{p}<2.55 \times 10^{-6}(0.05 / 19,645)$. The amount of inflation of observed versus expected $p$-values is given as the ratio of the median chi-squared statistics for observed to expected median p-values, referred to as Lambda $(\lambda)$. A conservative Bonferroni correction of multiple testing that assumes uncorrelated traits, would yield a similar $p$-value threshold of $p<2.30 \times 10^{-6}(0.05 / 23,004)$.

\section{PHESANT sensitivity analysis}

Analyses were re-run to assess residual confounding of assessment centre and genetic batch, including them as well as all 40 principal components as additional covariates for outcomes identified as strongly associated with either one of the disorders PRS. These covariates were not included in the first model because this 

by both genetic predisposition and outcomes, as discussed in the limitations section.

426 Furthermore, PRS were derived using various $p$-value thresholds $\left(p<0.01, p<0.1 \times 10^{-3}\right.$, $427 \mathrm{p}<1 \times 10^{-4}, \mathrm{p}<1 \times 10^{-5}, \mathrm{p}<1 \times 10^{-6}$ ) with consequently increasing numbers of SNPs (Table 428 S6) and the five MR-pheWAS were re-run with the more relaxed PRS to capture a 429 larger amount of explained variation in the disorders by accepting an increase in 430 horizontal pleiotropic effects. For MDD GWAS results were available for only 10,000 431 SNPs at these additional thresholds due to availability restrictions.

432 All analyses were performed in $\mathrm{R}$ version 3.2.4 ATLAS and $\mathrm{R}$ version 3.3.1, and the 433 code is available at [https://github.com/MRCIEU/Psychiatric-disorder-pheWAS434 UKBB]. Git tag v0.1 corresponds to the version presented here. 
435

436

437

438

LR
Data curation, Formal analysis, Investigation, Methodology, Software, Visualization, Writing - original draft, Writing - review \& editing

LACM Methodology, Software, Writing - review \& editing

Writing - review \& editing

Methodology, Writing - review \& editing

Conceptualization, Investigation, Funding acquisition, Writing-review \&

\section{FUNDING}

455 BL and LR are supported by the Wellcome Trust (grant ref: 204895/Z/16/Z) awarded 456 to AT, GDS, ES and KT. BL, LACM, GDS, KT, EW and ES work in a unit that receives 457 funding from the University of Bristol and the UK Medical Research Council 
458 (MC_UU_00011/1 and MC_UU_00011/3). LACM is funded by a University of Bristol

459 Vice-Chancellor's Fellowship.

460 CONFLICT OF INTEREST

461 The authors declare no conflict of interest. 
463 1. Polderman TJC, Benyamin B, de Leeuw CA, Sullivan PF, van Bochoven A, Visscher 464 PM, et al. Meta-analysis of the heritability of human traits based on fifty years of twin studies. 465 Nat Genet. 2015;47:702.

4662 2. Demontis D, Walters RK, Martin J, Mattheisen M, Als TD, Agerbo E, et al. Discovery 467 of the first genome-wide significant risk loci for attention deficit/hyperactivity disorder. Nat 468 Genet. 2019;51(1):63-75.

469 3. Ripke S, Consortium SWGotPG. Biological insights from 108 schizophrenia470 associated genetic loci. Nature. 2014;511(7510):421-7.

471 4. Grove J, Ripke S, Als TD, Mattheisen M, Walters RK, Won H, et al. Identification of common genetic risk variants for autism spectrum disorder. Nat Genet. 2019;51(3):431-44.

473 5. Wray NR, Ripke S, Mattheisen M, Trzaskowski M, Byrne EM, Abdellaoui A, et al. 474 Genome-wide association analyses identify 44 risk variants and refine the genetic architecture 475 of major depression. Nat Genet. 2018;50(5):668-81.

476 6. Thapar A, Cooper M, Rutter M. Neurodevelopmental disorders. Lancet Psychiatry. 477 2017;4(4):339-46.

478 7. Anttila V, Bulik-Sullivan B, Finucane HK, Walters RK, Bras J, Duncan L, et al. Analysis 479 of shared heritability in common disorders of the brain. Science. 2018;360(6395).

480 8. Wray NR, Lee SH, Mehta D, Vinkhuyzen AAE, Dudbridge F, Middeldorp CM. Research 481 Review: Polygenic methods and their application to psychiatric traits. J Child Psychol 482 Psychiatry. 2014;55(10):1068-87.

483 9. Fry A, Littlejohns TJ, Sudlow C, Doherty N, Adamska L, Sprosen T, et al. Comparison 484 of Sociodemographic and Health-Related Characteristics of UK Biobank Participants With 485 Those of the General Population. Am J Epidemiol. 2017;186(9):1026-34.

486 10. Richardson TG, Harrison S, Hemani G, Davey Smith G. An atlas of polygenic risk score 487 associations to highlight putative causal relationships across the human phenome. eLife. $488 \quad 2019 ; 8$.

489 11. Leppert B, Havdahl A, Riglin L, Jones HJ, Zheng J, Davey Smith G, et al. Association 490 of Maternal Neurodevelopmental Risk Alleles With Early-Life Exposures. JAMA Psychiatry. 491 2019.Epub 01May 2019

492 12. Loe IM, Feldman HM. Academic and Educational Outcomes of Children With ADHD. 493 J Pediat Psychol. 2007;32(6):643-54.

494 13. Stergiakouli E, Martin J, Hamshere ML, Heron J, St Pourcain B, Timpson NJ, et al. 495 Association between polygenic risk scores for attention-deficit hyperactivity disorder and 
educational and cognitive outcomes in the general population. Int $\mathrm{J}$ Epidemiol. 2017;46(2):421-8.

14. Rhodes JD, Pelham WE, Gnagy EM, Shiffman S, Derefinko KJ, Molina BS. Cigarette smoking and ADHD: An examination of prognostically relevant smoking behaviors among adolescents and young adults. Psychol Addict Behav. 2016;30(5):588-600.

15. Flory K, Molina BS, Pelham WE, Jr., Gnagy E, Smith B. Childhood ADHD predicts risky sexual behavior in young adulthood. J Clin Child Adoles Psychol. 2006;35(4):571-7.

16. Hanc T, Cortese S. Attention deficit/hyperactivity-disorder and obesity: A review and model of current hypotheses explaining their comorbidity. Neurosci Biobehav Rev. 2018;92:16-28.

17. Neumeyer AM, Cano Sokoloff N, McDonnell El, Macklin EA, McDougle CJ, Holmes TM, et al. Nutrition and Bone Density in Boys with Autism Spectrum Disorder. J Acad Nutr Diet. 2018;118(5):865-77.

18. Ekhlaspour L, Baskaran C, Campoverde KJ, Sokoloff NC, Neumeyer AM, Misra M. Bone Density in Adolescents and Young Adults with Autism Spectrum Disorders. J Autism Dev Disord. 2016;46(11):3387-91.

19. Neumeyer AM, O'Rourke JA, Massa A, Lee H, Lawson EA, McDougle CJ, et al. Brief report: bone fractures in children and adults with autism spectrum disorders. J Autism Dev Disord. 2015;45(3):881-7.

20. Mostafa GA, Al-Ayadhi LY. Reduced serum concentrations of 25-hydroxy vitamin D in children with autism: relation to autoimmunity. J Neuroinflamm. 2012;9:201.

21. Macova L, Bicikova M, Ostatnikova D, Hill M, Starka L. Vitamin D, neurosteroids and autism. Physiol Res. 2017;66(Supplementum 3):S333-s40.

22. van der Merwe C, Passchier R, Mufford M, Ramesar R, Dalvie S, Stein DJ. Polygenic risk for schizophrenia and associated brain structural changes: A systematic review. Compr Psychiatry. 2019;88:77-82.

23. Gao X, Mi Y, Guo N, Xu H, Jiang P, Zhang R, et al. Glioma in Schizophrenia: Is the Risk Higher or Lower? Front Cell Neurosci. 2018;12:289.

24. van Erp TG, Hibar DP, Rasmussen JM, Glahn DC, Pearlson GD, Andreassen OA, et al. Subcortical brain volume abnormalities in 2028 individuals with schizophrenia and 2540 healthy controls via the ENIGMA consortium. Mol Psychiatry. 2016;21(4):585.

25. Okada N, Fukunaga M, Yamashita F, Koshiyama D, Yamamori H, Ohi K, et al. Abnormal asymmetries in subcortical brain volume in schizophrenia. Mol Psychiatry. 2016;21(10):1460-6.

26. Franke B, Stein JL, Ripke S, Anttila V, Hibar DP, van Hulzen KJE, et al. Genetic influences on schizophrenia and subcortical brain volumes: large-scale proof of concept. Nat Neurosci. 2016;19(3):420-31. 
27. Hughes RA, Davies NM, Davey Smith G, Tilling K. Selection bias in instrumental variable analyses. bioRxiv. 2018:192237.

28. Reitan R. Validity of the Trail Making Test as an indicator of organic brain damage. Percept and Mot Skills. 1958(8):271-6.

29. Aleman A, Hijman R, de Haan EH, Kahn RS. Memory impairment in schizophrenia: a meta-analysis. Am J Psychiatry. 1999;156(9):1358-66.

30. Wolwer W, Gaebel W. Impaired Trail-Making Test-B performance in patients with acute schizophrenia is related to inefficient sequencing of planning and acting. J Psychiatr Res. 2002;36(6):407-16.

31. Heinrichs RW, Zakzanis KK. Neurocognitive deficit in schizophrenia: a quantitative review of the evidence. Neuropsychology. 1998;12(3):426-45.

32. Zalla T, Joyce C, Szoke A, Schurhoff F, Pillon B, Komano O, et al. Executive dysfunctions as potential markers of familial vulnerability to bipolar disorder and schizophrenia. Psychiatry Res. 2004;121(3):207-17.

33. Sitskoorn MM, Aleman A, Ebisch SJ, Appels MC, Kahn RS. Cognitive deficits in relatives of patients with schizophrenia: a meta-analysis. Schizophr Res. 2004;71(2-3):28595.

34. Perianez JA, Rios-Lago M, Rodriguez-Sanchez JM, Adrover-Roig D, Sanchez-Cubillo I, Crespo-Facorro B, et al. Trail Making Test in traumatic brain injury, schizophrenia, and normal ageing: sample comparisons and normative data. Arch Clin Neuropsychol. 2007;22(4):433-47.

35. Zhou RY, Wang JJ, Sun JC, You Y, Ying JN, Han XM. Attention deficit hyperactivity disorder may be a highly inflammation and immune-associated disease (Review). Mol Med Rep. 2017;16(4):5071-7.

36. Galecki P, Talarowska M. Inflammatory theory of depression. Psychiatr Pol. 2018;52(3):437-47.

37. Savitz J, Harrison NA. Interoception and Inflammation in Psychiatric Disorders. Biol Psychiatry Cogn Neurosci Neuroimaging. 2018;3(6):514-24.

38. Tonacci A, Billeci L, Ruta L, Tartarisco G, Pioggia G, Gangemi S. A systematic review of the association between allergic asthma and autism. Minerva Pediatr. 2017;69(6):538-50. 39. Billeci L, Tonacci A, Tartarisco G, Ruta L, Pioggia G, Gangemi S. Association Between Atopic Dermatitis and Autism Spectrum Disorders: A Systematic Review. Am J Clin Dermatol. 2015;16(5):371-88.

40. Schans JV, Cicek R, de Vries TW, Hak E, Hoekstra PJ. Association of atopic diseases and attention-deficit/hyperactivity disorder: A systematic review and meta-analyses. Neurosci Biobehav Rev. 2017;74(Pt A):139-48. 
569 41. Taylor AE, Jones HJ, Sallis H, Euesden J, Stergiakouli E, Davies NM, et al. Exploring

570 the association of genetic factors with participation in the Avon Longitudinal Study of Parents

571 and Children. Int J Epidemiol. 2018.

572 42. Munafò MR, Tilling K, Taylor AE, Evans DM, Davey Smith G. Collider scope: when 573 selection bias can substantially influence observed associations. Int $\mathrm{J}$ Epidemiol. 574 2018;47(1):226-35.

575 43. Lawlor DA, Tilling K, Davey Smith G. Triangulation in aetiological epidemiology. Int J 576 Epidemiol. 2016;45(6):1866-86.

577 44. Davey Smith G, Ebrahim S. Epidemiology--is it time to call it a day? Int J Epidemiol. $578 \quad 2001 ; 30(1): 1-11$.

579 45. Mitchell R, Elsworth B, Raistrick C, Paternoster L, Hemani G, Gaunt T. MRC IEU UK

580 Biobank GWAS pipeline version 2.

581 https://doi.org/10.5523/bris.pnoat8cxo0u52p6ynfaekeigi2019.

582 46. Martin J, Taylor MJ, Rydell M, Riglin L, Eyre O, Lu Y, et al. Sex-specific manifestation

583 of genetic risk for attention deficit hyperactivity disorder in the general population. J Child 584 Psychol Psychiatry. 2018.

585 47. Ruderfer DM, Ripke S, McQuillin A, Boocock J, Stahl EA, Pavlides JMW, et al. 586 Genomic Dissection of Bipolar Disorder and Schizophrenia, Including 28 Subphenotypes. 587 Cell. 2018;173(7):1705-15.e16.

588 48. Millard LAC, Davies NM, Gaunt TR, Davey Smith G, Tilling K. Software Application 589 Profile: PHESANT: a tool for performing automated phenome scans in UK Biobank. Int $J$ 590 Epidemiol. 2017.

591 49. Millard LAC, Davies NM, Tilling K, Gaunt TR, Davey Smith G. Searching for the causal 592 effects of body mass index in over 300000 participants in UK Biobank, using Mendelian 593 randomization. PLoS genetics. 2019;15(2):e1007951.

594 50. Zheng J, Richardson TG, Millard LAC, Hemani G, Elsworth BL, Raistrick CA, et al. 595 PhenoSpD: an integrated toolkit for phenotypic correlation estimation and multiple testing 596 correction using GWAS summary statistics. GigaScience. 2018;7(8). 
Figure 1. Study overview. (A) Descriptive sample overview of selected outcomes in

UK Biobank. (B) Categories of UK Biobank with the size of pie chart sections indicating the number of included outcomes: socio-demographics $(n=2,057)$, general health $(n=19,740)$, mental health $(n=233)$, brain and cognition $(n=974)$.

Figure 2. Overview of the distribution of disorder specific polygenic risk scores $\left(p<5 \times 10^{-8}\right)$ associated outcomes per category of the UK Biobank variables catalogue. Shown are the number of associations with polygenic risk scores for attention deficit/hyperactivity disorder (ADHD), autism spectrum disorder (ASD), schizophrenia (SCZ), major depression (MDD) and bipolar disorder (BP).

Figure 3. MR-PheWAS results for attention deficit/hyperactivity disorder (ADHD), autism spectrum disorder (ASD), schizophrenia (SCZ), major depressive disorder (MDD) and bipolar disorder (BP). Left hand panel: $Q Q$ plots of expected versus observed $p$-values for association of PRS with all outcomes in UK Biobank. Red line indicates the significance threshold $\left(2.5 \times 10^{-6}\right)$. Lambda $(\lambda)$ indicates the degree of inflation from the expected fit. Right hand panel: selected results from different categories with $p$-values below the significance threshold and estimates generated by PHESANT. Results for continuous outcomes (std. $\beta$ ) are the standard deviation change of inverse-rank normal transformed outcome per 1 SD higher PRS.

Figure 4. Cross-disorder comparison. Shown are standardized log odds (upper section in each panel) or standardized beta-values (lower section of each panel) of all outcomes associated with polygenic risk scores for either attention deficit/hyperactivity disorder (ADHD), autism spectrum disorder (ASD), schizophrenia (SCZ), major depressive disorder (MDD) or bipolar disorder (BP) at $p<2.55 \times 10^{-6}$ as indicated by 
stars $\left(^{*}\right)$. For outcomes categorized as ordered-logistic, only one outcome is displayed.

624 Only associations with anthropometric measures of the right side of the body are shown. Estimates were generated by PHESANT. Results for continuous outcomes (std. beta) are the standard deviation change of inverse-rank normal transformed outcome per 1 SD higher PRS.

Figure 5. Categories of highly associated outcomes with polygenic risk scores for attention deficit/hyperactivity disorder (ADHD), autism spectrum disorder (ASD), schizophrenia (SCZ), major depressive disorder (MDD) and bipolar disorder (BP). Size of categories depends on the relative number of associated outcomes to the total number of outcomes within each category. Only categories with more than 1 variables are shown. Lifestyle and socio-demographic factors are shown in orange, physical health measures are shown in green and mental health, brain and cognition traits are shown in violet. Grey categories had zero hits for the corresponding disorder.

Figure 6. Overview of study sample derivation. Participants with withdrawn consent, sex mismatch or sex aneuploidy where already removed from the dataset in standard 


\section{SUPPORTING INFORMATION}

641

642 Figure S1. Overview of the distribution of disorder specific polygenic risk scores $\left(p<5 \times 10^{-8}\right)$ associated outcomes per category of the UK Biobank variables catalogue after FDR adjustment for multiple testing. Shown are the number of associations with polygenic risk scores for attention deficit/hyperactivity disorder (ADHD), autism spectrum disorder (ASD), bipolar disorder (BP), major depressive disorder (MDD) and schizophrenia (SCZ).

Figure S2. Top associated outcomes with PRS for attention deficit/hyperactivity disorder (ADHD), autism spectrum disorder (ASD), schizophrenia (SCZ), bipolar disorder (BP) and major depressive disorder (MDD) across different p-value thresholds for SNP inclusion (5x10-8 - 1x10-2).

Table S1. Overview of UK Biobank categories with total number of outcomes per category and number of associated outcomes with polygenic risk scores passing the significance threshold $\left(p<2.55 \times 10^{-6}\right)$. (ADHD- attention defict/ hyperactivity disorder, ASD- autism spectrum disorder, SCZ- schizophrenia, MDD- major depressive disorder, BP- bipolar disorder)

Table S2. Correlation matrix of polygenic risk scores $\left(p<5 \times 10^{-8}\right)$. Correlation coefficients are displayed on the left side, $p$-values on the right side of the table. (ADHD- attention defict/ hyperactivity disorder, ASD- autism spectrum disorder, SCZschizophrenia, MDD- major depressive disorder, BP- bipolar disorder)

Table S3. MR-PheWAS results for association of generic risk of 5 common psychiatric disorders with 23,004 outcomes in UK Biobank. Genetic risk is defined as weighted sum of all genome-wide significant risk alleles for each disorder in 334,976 participants 
664

665

666

667

668

669

670

671

672

673

674

675

676

677

678

679

of the UK Biobank. Estimates were generated by PHESANT. Results for continuous outcomes are the standard deviation change of inverse-rank normal transformed outcome per 1 SD higher PRS.

Table S4. MR-PheWAS follow-up and sensitivity results for selected continuous outcomes. Genetic risk is defined as weighted sum of all genome-wide significant risk alleles for each disorder in 334,976 participants of the UK Biobank. Estimates were generated by linear regression on the original variable scale per 1 SD higher PRS.

Table S5. Number of strongly associated traits with PRS for attentiondeficit/hyperactivity disorder (ADHD), autism spectrum disorder (ASD), schizophrenina (SCZ), major depressive disorder (MDD) and bipolar disorder (BP) at different $p$-value thresholds for PRS calculation.

Table S6. Number of SNPs included in polygenic risk scores for attentiondeficit/hyperactivity disorder (ADHD), autism spectrum disorder (ASD), schizophrenina (SCZ), major depressive disorder (MDD) and bipolar disorder (BP) at different p-value thresholds.

Text S1. Sensitivity analysis for autism spectrum disorder

Text S2. UK Biobank outcomes description 


\section{A}

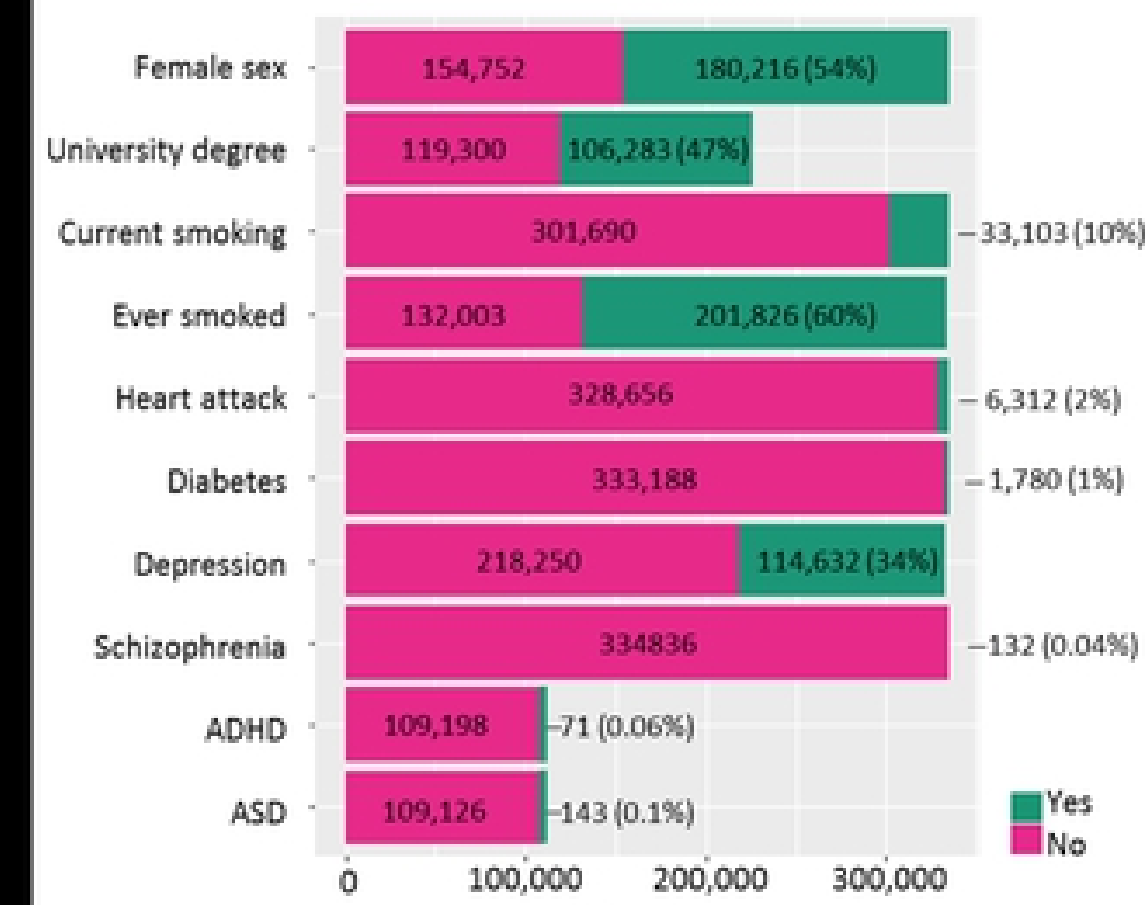
Number of participants

\section{B Socio-demographics (9\%)}

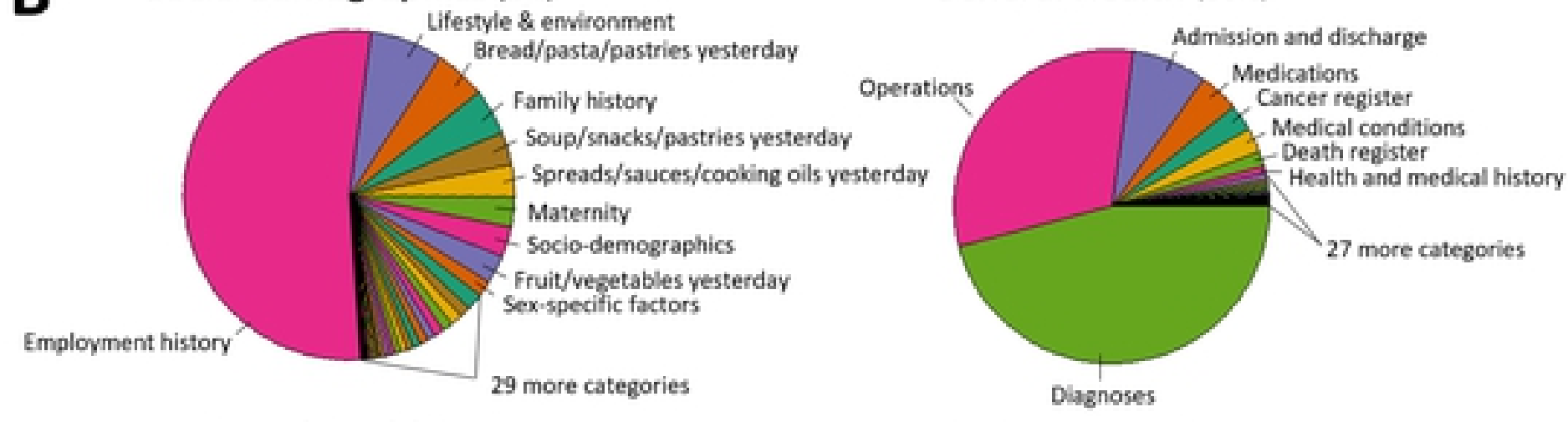

Mental Health (1\%)

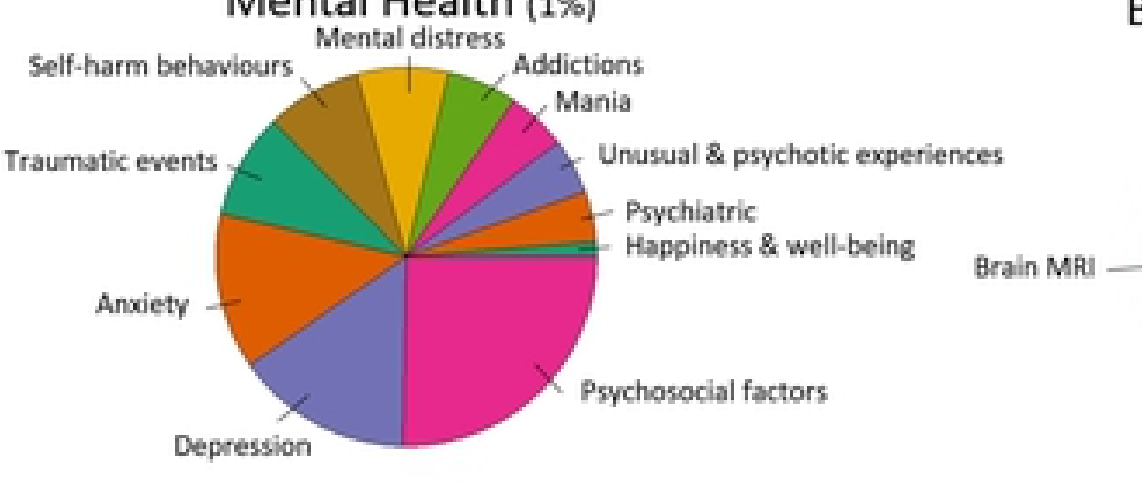

Brain \& Cognition (4\%)

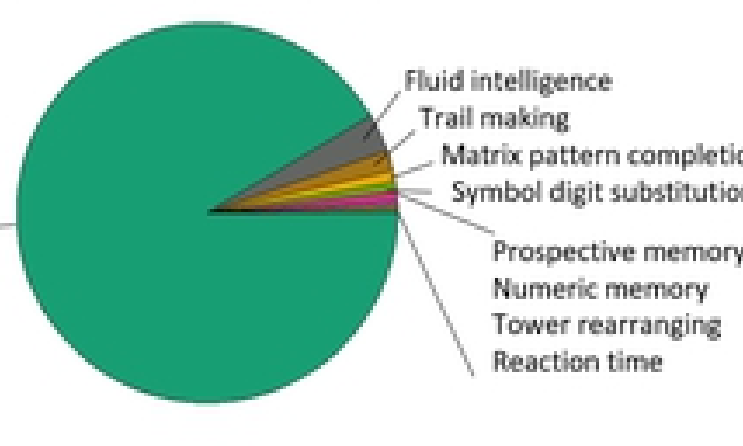

Figure 1 
Lifestyle and environmentAlcohol and Cannabis -

Sociodemographics -

Sex-specific factors -

Family history-

Early life factors -

Baseline characteristics -

Work environment -

Trail making -

Brain MRI-

Fluid intelligencePsychosocial factors -

Mental distress -

Traumatic events -

DepressionAnxietyMania-

Happiness and well-beeing -

Self-harm behaviors -

Anthropometry Health and medical history-

DiagnosesBlood assays Operations Medications Bone-densitometry of heelCancer register Medical conditions Spirometry Urine assays Admission and discharge-

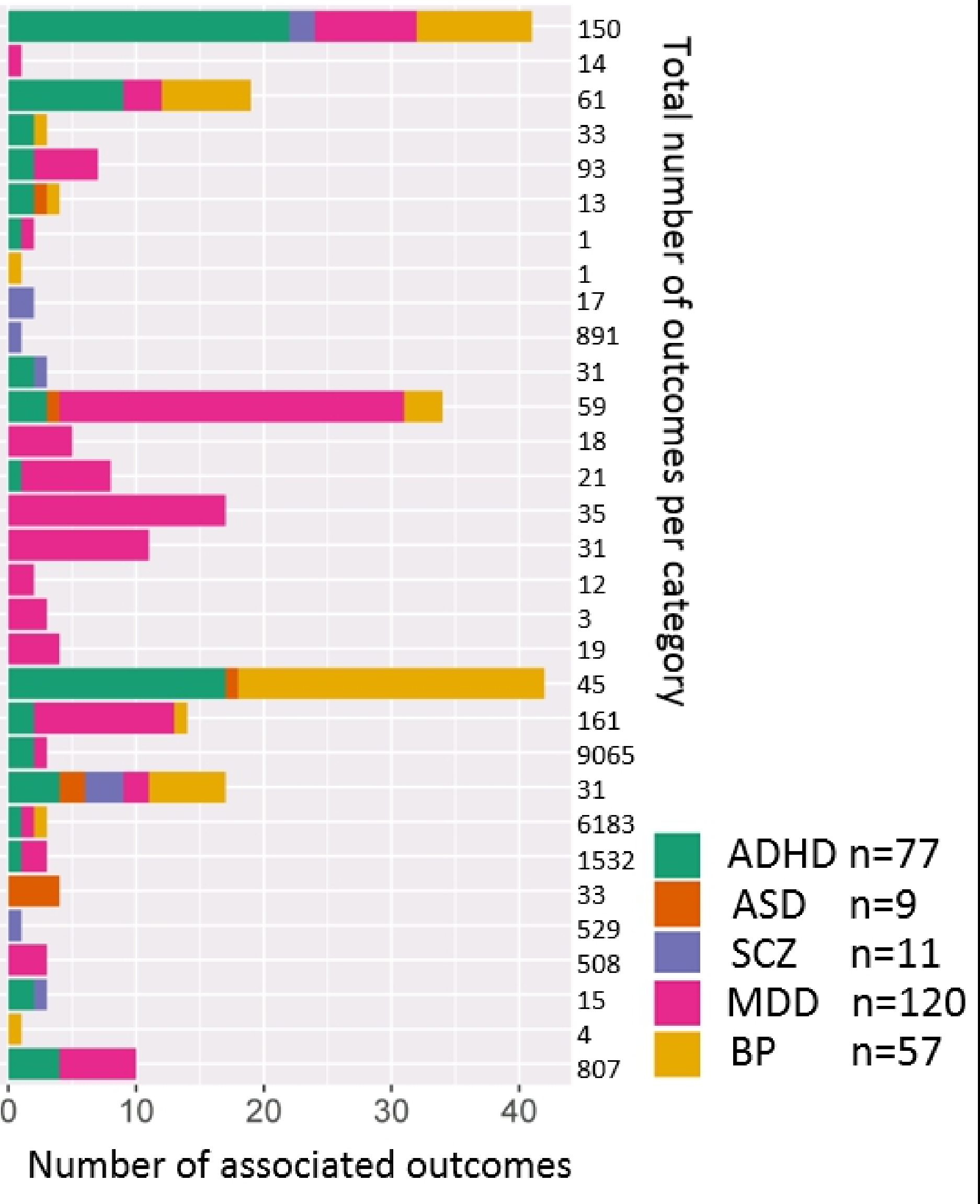

Figure 2 


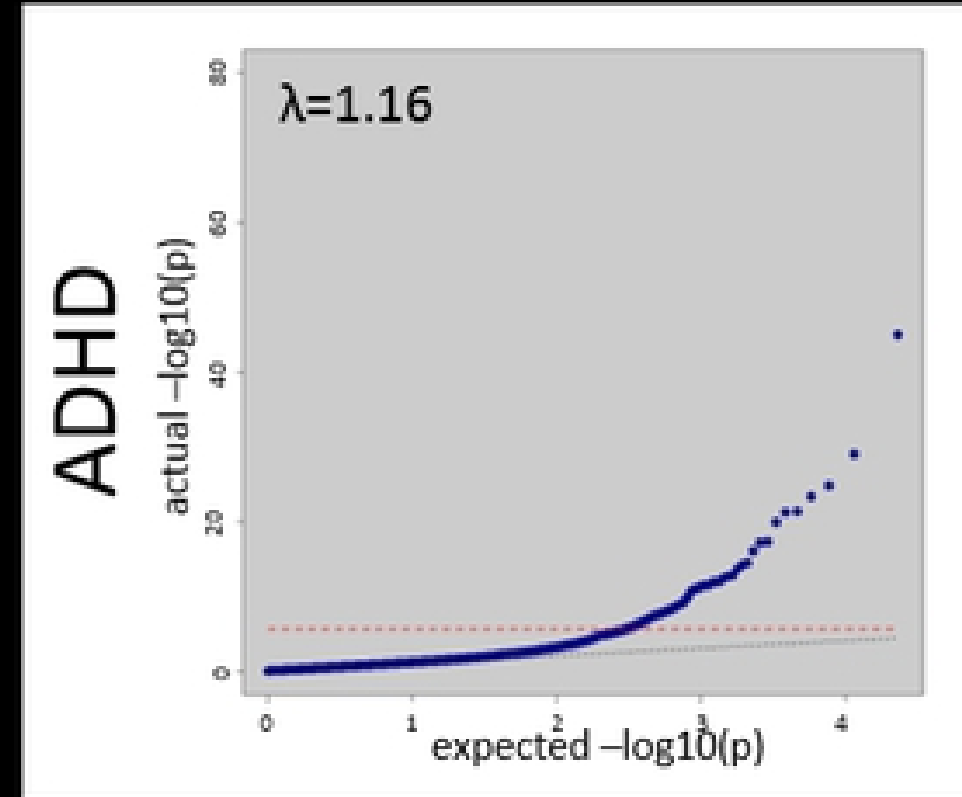

std. beta/log odds* $(95 \% \mathrm{Cl})$
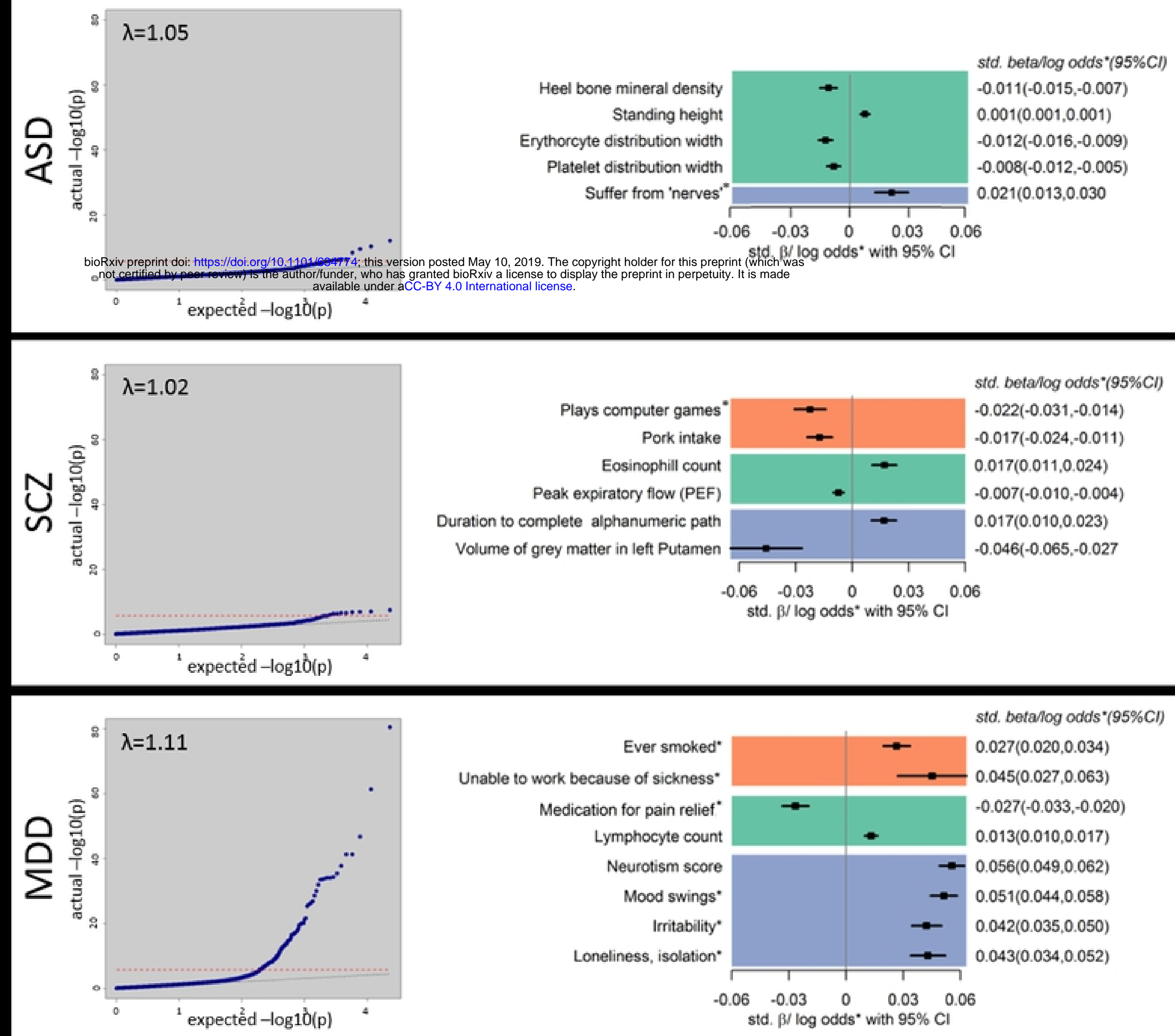

std. beta/log odds * $(95 \% \mathrm{Cl})$

requency of unenthusiasm in last 2 weeks

Physically abused by family as a child

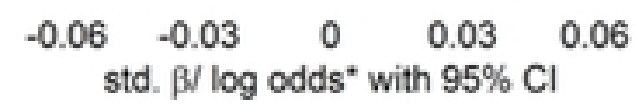

$-0.025(-0.029,-0.022)$

$-0.009(-0.013,-0.006)$

$-0.043(-0.050,-0.035)$

$-0.031(-0.038,-0.025)$

$0.045(0.034,0.057)$

$0.011(0.008,0.015)$

$0.028(0.028,0.019)$

$0.038(0.023,0.054)$

std. $\beta / \log$ odds* with $95 \% \mathrm{Cl}$

std. beta/log odds* $(95 \% \mathrm{Cl})$

$-0.022(-0.031,-0.014)$

$-0.007(-0.010,-0.004)$

$0.017(0.010,0.023)$

std. $\beta / \log$ odds* with $95 \% \mathrm{Cl}$
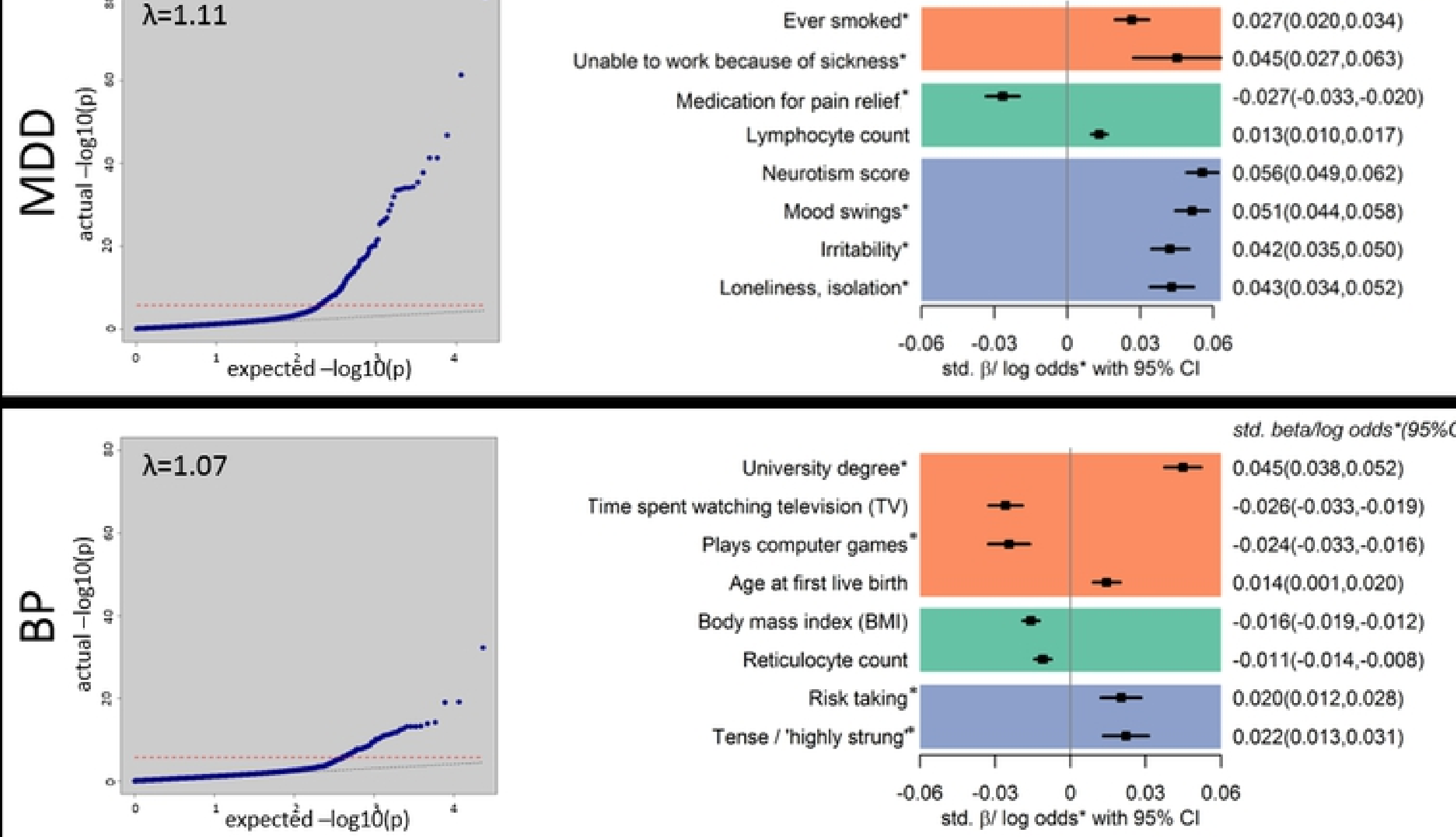

std. beta/log odds* $(95 \% \mathrm{Cl})$

$0.045(0.038,0.052)$

$-0.026(-0.033,-0.019)$

$-0.024(-0.033,-0.016)$

$0.014(0.001,0.020)$

$-0.016(-0.019,-0.012)$

$-0.011(-0.014,-0.008)$

$0.020(0.012,0.028)$

$0.022(0.013,0.031)$

Socio-demographic factors General health

Mental health and cognition

\section{Figure 3}




\section{Socio-demographics}

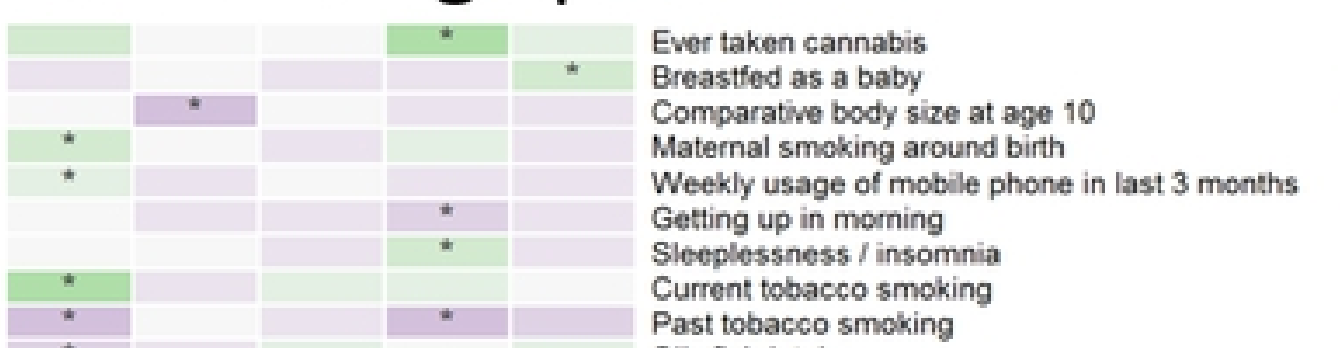

Past tobacco smoking

bioRxiv preprint doi: https://doi.org/10.1109/634 ff 4 inthis version posted May 10, 2019. The copyright holder for this preprint (which was orlfendere who has granted bioRxiv a license to display the preprint in perpetuity. It is made Pavailablehunder ad

Alcohol intake frequency

Alcohol usually taken with meals

Alcohol intake versus 10 years previously

Facial ageing

Ever smoked

Plays computer games

Frequency of stair climbing in last 4 weeks

University degree

Unable to work because of sickness

Average total household income before tax

Job involves heavy manual or physical work

Townsend deprivation index at recruitment Mother's age

Father's age

Time spend outdoors in summer

Time spent watching television (TV)

Dried fruit intake

Average weekly red wine intake

Pack years of smoking

Pack years adult smoking as proportion of life span

Age first had sexual intercourse

Lifetime number of sexual partners

Frequency of solarium/sunlamp use

Length of time at current address

Age completed full time education

\section{General health}

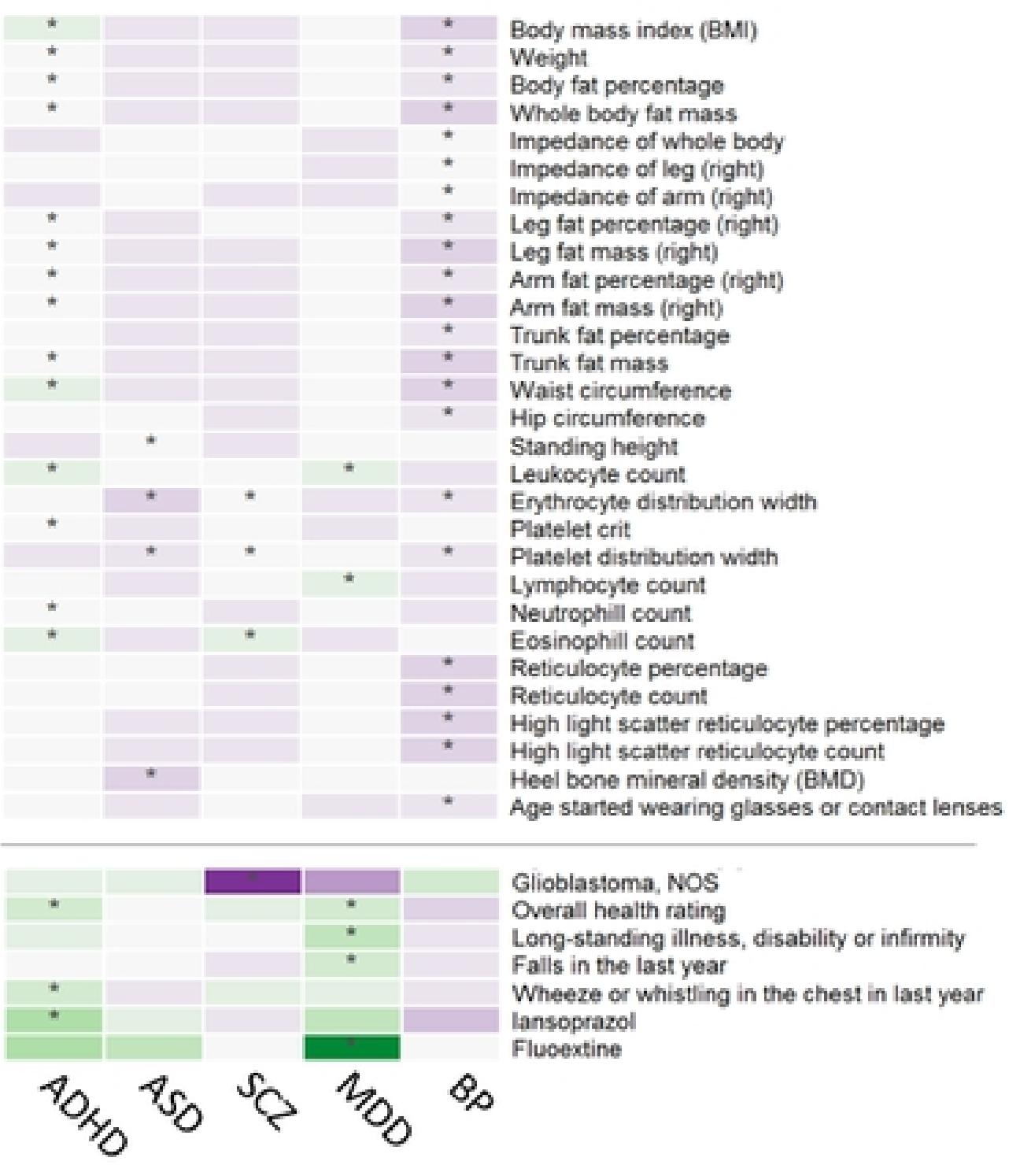

\section{Mental health}
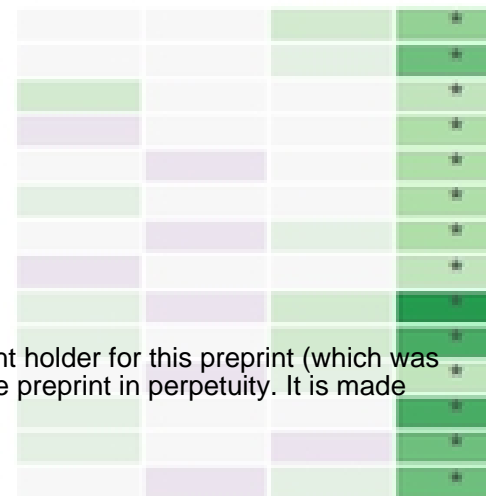

$+$

Feelings of worthlessness during worst period of depression Recent feelings of inadequacy

Recent trouble concentrating on things

Recent feelings of depression

Recent poor appetite or overeating

Recent lack of interest or pleasure in doing things

Trouble falling or staying asleep, or sleeping too much

Recent feelings of tiredness or low energy

Activities undertaken to treat depression

Ever had period extreme irritability

Manifestations of mania or irritability

Bulimia nervosa diagnosed

Panic attacks diagnosed

Mood swings

Miserablenes:

Imitability

Sensitivity / hurt feelings

Fed-up feelings

Nervous feelings

Worrier / anxious feelings

Tense / 'highly strung'

Worry too long after embarrassment

Suffer from 'nerves'

Loneliness, isolation

Guilty feelings

Risk taking

Frequency of depressed mood in last 2 weeks

Frequency of unenthusiasm / disinterest in last 2 weeks

Frequency of tenseness / restlessness in last 2 weeks

Frequency of tiredness / lethargy in last 2 weeks

Seen doctor (GP) for nerves, anxiety, tension or depression

Seen a psychiatrist for nerves, anwiety, tension or depression Happiness

Health satisfaction

Friendships satisfaction

Ever depressed for a whole week

Ever depressed for a whole weex

Ever unenthusiasticidisinterested for a whole week

Ever manic/hyper for 2 days

Illness, injury, bereavement, stress in last 2 years

Leisure/social activities

Leisure/social activities

Felt hated by family member as a child

Physically abused by family as a child

Felt loved as a child

Felt loved as a child

inations because of provious stressful experience Repeated disturbing thoughts of stressful experience in past month Felt very upset when reminded of stressful experience in past month

Lifetime number of depressed periods

Neuroticism score

\section{Brain \& cognition}

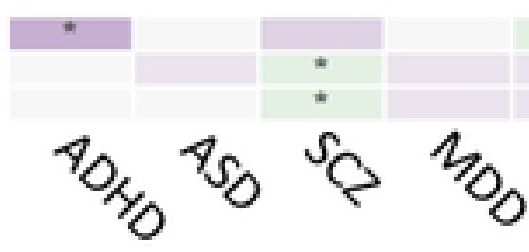

Fluid intelligence score

Interval between previous point and current one in alphanumeric path

Duration to complete alphanumeric path (trail $* 2$ )

\section{\&s}

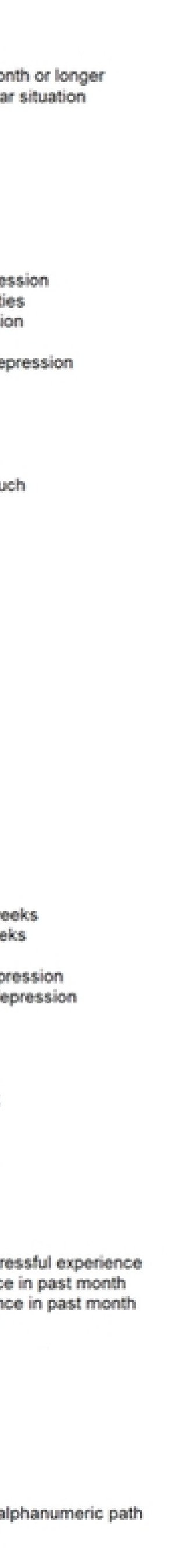

\section{Figure 4}


ADHD

Lifestyle\&environment Sociodemographics Anthropometry

is islood assays

Spirometry

Co Trummatc events

选

MDD

Bone dencensinsitometry Early-life factors

Depression

BP

SCZ

Blood assays Trail making

Fluid intelligence

के

Blood assays

Anthropometry

Psychosocial factors

Traumatic events

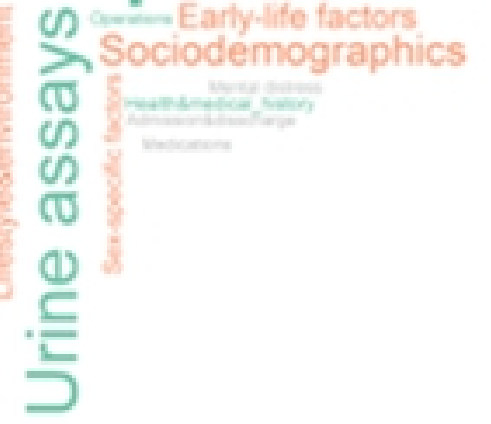

Figure 5 


\section{3,010 UK Biobank participants with genetic data after standard QC}

54,757 removed due to non-white British ancestry

73,277 removed due to relatedness

334,976 participants

Figure 6 